05

\title{
Влияние состава на атомную структуру образцов $\mathrm{Bi}_{1-x} \mathrm{Y}_{x} \mathrm{FeO}_{3}$
}

\author{
() 3.А. Самойленко, ${ }^{1}$ Н.Н. Ивахненко, ${ }^{1,2}$ Е.И. Пушенко, ${ }^{1}$ В.Я. Сычева, ${ }^{1}$ H.А. Леденев, ${ }^{3}$ А.В. Пащенко ${ }^{3}$ \\ ${ }^{1}$ Донецкий фозико-технический институт им. А.А. Галкина, \\ 83114 Донецк, Украина \\ ${ }^{2}$ Донецкий национальный университет экономики и торговли им. Михаила Туган-Барановского, \\ 83050 Донецк, Украина \\ ${ }^{3}$ Донецкий фризико-технический институт им. А.А. Галкина НАН Украины, \\ 03028, Киев, Украина \\ e-mail: yulduz19.77@mail.ru
}

Поступило в Редакцию 18 июля 2020 г.

В окончательной редакции 10 ноября 2020 г.

Принято к публикации 22 ноября 2020 г.

Проанализирована атомная структура в образцах $\mathrm{Bi}_{1-x} \mathrm{Y}_{x} \mathrm{FeO}_{3}$ от изменения концентрационного состава иттрия при $x=0,0.1,0.15,0.2$. Образцы получены методом быстрого жидкого спекания с помощью обычной реакции в твердом состоянии. В результате рентгеноструктурных исследований образцов $\mathrm{Bi}_{1-x} \mathrm{Y}_{x} \mathrm{FeO}_{3}$ с увеличением $x$ обнаружен концентрационный фазовый переход ромбоэдрической фазы в орторомбическую при $x=0.2$ и точке перегиба $x=0.15$. Показано, что с увеличением содержания иттрия относительные диэлектрические постоянные и тангенсы диэлектрических потерь образцов постепенно уменьшались.

Ключевые слова: мультиферроики, феррит висмута, концентрационный фазовый переход, диэлектрическая проницаемость, тангенс угла диэлектрических потерь.

DOI: $10.21883 /$ JTF.2021.05.50689.228-20

\section{Введение}

Возрастание интереса в последние годы к мультиферроикам вызвано тем, что они обладают двумя или более типами упорядочения. Мультиферроики, обладающие одновременно сегнетоэлектрическим и магнитным упорядочением, имеют возможность управления магнитными и электрическими свойствами. Среди перовскитов важное место занимает феррит висмута $\mathrm{BiFeO}_{3}$, который относится к высокотемпературным мультиферроикам $[1,2]$. Этот перовскит, в силу своих особенностей антиферромагнитного упорядочения спинов, обладает слабым ферромагнетизмом, а следовательно, слабым магнитоэлектрическим эффектом, что ограничивает его практическое использование. С целью уменьшения этого ограничения используют твердые растворы на основе феррита висмута [3,4]. Целью настоящей работы было исследование изменений в атомном порядке и диэлектрических параметров мультиферроика $\mathrm{Bi}_{1-x} \mathrm{Y}_{x} \mathrm{FeO}_{3}$ при $x=0,0.1,0.15,0.2$.

\section{1. Материал и методики эксперимента}

Керамические образцы $\mathrm{Bi}_{1-x} \mathrm{Y}_{x} \mathrm{FeO}_{3}$ с $x=0,0.1,0.15$, 0.2 были получены методом быстрого жидкого спекании (БЖС) [5]. Особенности получения феррита висмута методом БЖС опубликованы в [5,6]. Для дегидратации исходные порошки высокой чистоты $\mathrm{Bi}_{2} \mathrm{O}_{3}$ (99.5\%), $\mathrm{Y}_{2} \mathrm{O}_{3}(>99.5 \%)$ и $\mathrm{Fe}_{2} \mathrm{O}_{3}(>99.5 \%)$ прокаливались при температуре $180^{\circ} \mathrm{C}$ в течение $4 \mathrm{~h}$. Смесь исходных порошков в стехиометрическом соотношении измельчали в агатовой ступке, просеивали через сито $(40 \mu \mathrm{m})$ и прессовали в таблетки диаметром $5 \mathrm{~mm}$ под давлением $P=200 \mathrm{MPa}$. Полученные прессовки спекали на воздухе при температуре $t_{\mathrm{ann}}=850^{\circ} \mathrm{C}$ в течение $480 \mathrm{~s}$ в режиме быстрого нагрева и охлаждения. Нагрев проводили со скоростью $100^{\circ} \mathrm{C} / 10 \mathrm{~s}$ в температурном интервале от комнатной температуры до $770^{\circ} \mathrm{C}$ и со скоростью $10^{\circ} \mathrm{C} / \mathrm{min}$ в интервале от 770 до $850^{\circ} \mathrm{C}$. Охлаждали образцы от $850^{\circ} \mathrm{C}$ до комнатной температуры со скоростью $100^{\circ} \mathrm{C} / 10 \mathrm{~s}$. В настоящнй работе использовали метод рентгеновской дифракции с помощью регистрации дифракционной картины на рентгеновскую пленку с последующим микрофотометрированием дебаеграмм, что увеличивало чувствительность анализа образцов с повышенной внутренней дефектностью кристаллографической многокомпонентной атомной структуры [7]. Измерения диэлектрических параметров $\mathrm{Bi}_{1-x} \mathrm{Y}_{x} \mathrm{FeO}_{3}$, a именно диэлектрической проницаемости и тангенса угла диэлектрических потерь, были проведены при комнатной температуре в низкочастотном (НЧ) диапазоне от 1 до $10^{6} \mathrm{~Hz}$ емкостным методом и в сверхвысокочастотном (СВЧ) диапазоне от 8 до $12 \mathrm{GHz}$ методом составного диэлектрического резонатора [8].

\section{2. Результаты эксперимента и обсуждение}

Исследование атомной структуры в образцах $\mathrm{Bi}_{1-x} \mathrm{Y}_{x} \mathrm{FeO}_{3}$ при $x=0,0.1,0.15,0.2$ проведено в результате анализа дифракционных картин (рис. 1-4). На приведенных картинах от образцов видно, что 


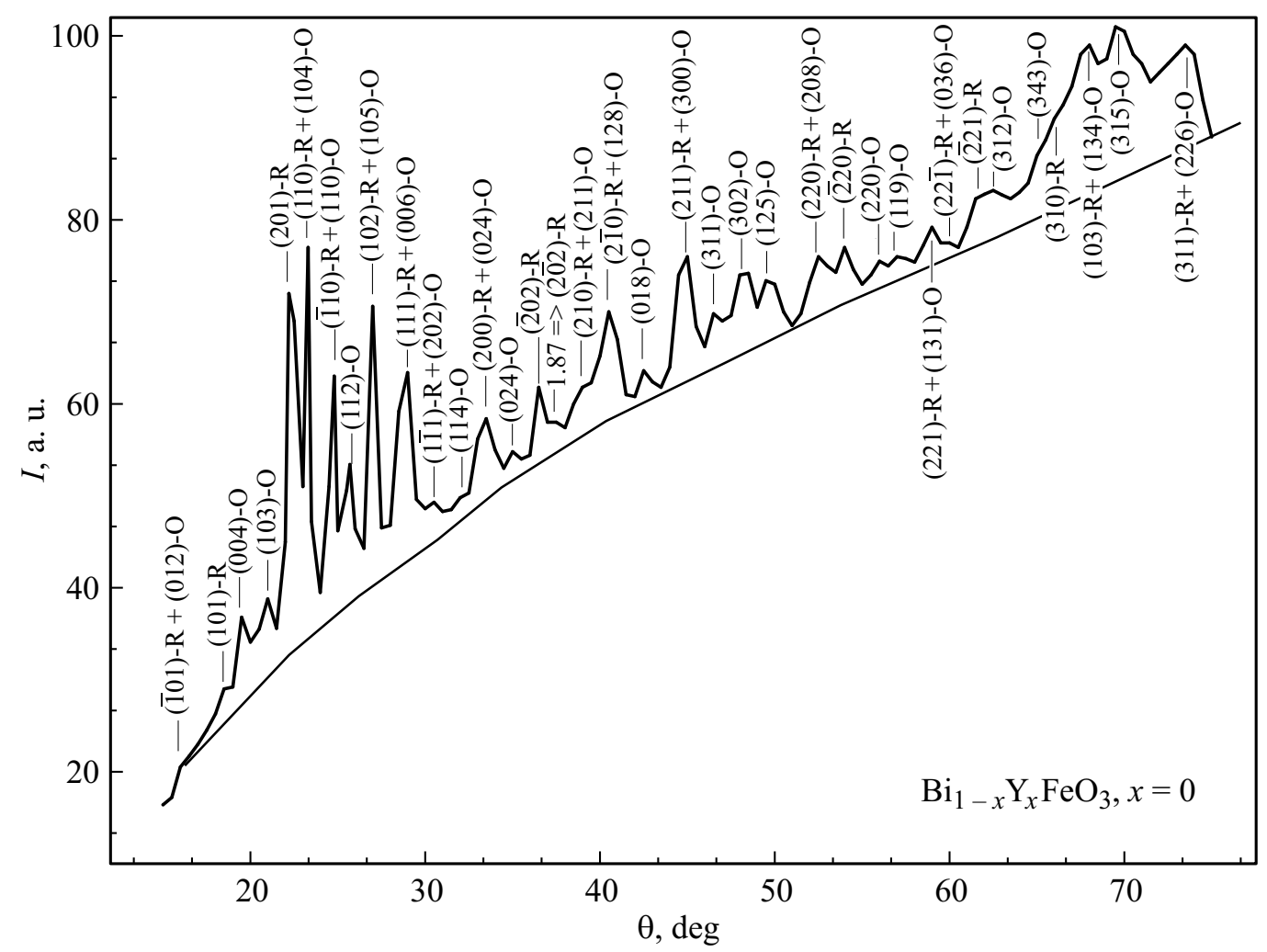

Рис. 1. Дифракционная картина от образца $\mathrm{Bi}_{1-x} \mathrm{Y}_{x} \mathrm{FeO}_{3}$ при $x=0$ (13ī).

дифракционные картины представляют суперпозицию рассеяния рентгеновских лучей от атомных группировок с различным масштабом упорядочения, а именно присутствуют узкие дебаевские линии; широкие диффузные максимумы когерентного рассеяния [9] от плоскостей с намечающимся дальним порядком наноразмерного масштаба в расположении атомов; диффузные полосы, полученные в результате когерентного рассеяния от мелких группировок атомов аморфизированной кластеризованной структуры с дальним и наноразмерным атомным порядком, и фон, возникающий в результате некогерентного диффузного рассеяния рентгеновского излучения от разупорядоченно расположенных атомов, с ближним атомным порядком, свойственным конденсированному состоянию данного материала.

На рис. 1 представлена дифракционная картина от приготовленного по описанной методике (БЖС) образца $\mathrm{Bi}_{1-x} \mathrm{Y}_{x} \mathrm{FeO}_{3}$ при $x=0$, форма записи которого целесообразна в данном случае, поскольку при известном составе $\mathrm{BiFeO}_{3}$ велика гибкость структуры этих материалов на внешнее воздействие, каким в нашем случае является способ приготовления.

Из рис. 1 видно, что самая яркая линия представляет собой суммарный максимум двух фаз (110)-R (R - ромбоэдрическая) и (104)-О (О - орторомбическая). Рядом с этой линией расположена интенсивная группа из трех линий (10)- $\mathrm{R}+(110)-\mathrm{O},(112)-\mathrm{O},(102)-\mathrm{R}+(105)-\mathrm{O})$, что указывает на взаимодействие атомов в соседних кри- сталлографических структурах, вызвавших локальную аморфизацию перечисленных групп плоскостей, и проявляется в форме диффузного гало у подошвы перечисленных дифракционных линий, а также и в других углах дифракционной картины, например, (21̄0)-R+(128)-O, (103)-R+(134)-O, (311)-R+(226)-O.

При введении в состав керамики иттрия в концентрации $x=0.1$ на дифракционной картине (рис. 2) основной интерференционный максимум также принадлежит двум фазам (110)-R и (104)-O.

При этом рядом с этим максимумом расположена группа из линий (110)-R+(110)-O, (112)-O, (102)-R+(105)-O, (111)-R+(006)-O), основания которых перекрылись в виде гало, свидетельствующем об аморфизации в данной структурной группе плоскостей; подобная локальная аморфизация произошла и в большеугловой группе плоскостей, а именно (310)-R+(128)-O, (103)-R+(134)-O, (311)-R+(226)-O.

$\mathrm{C}$ увеличением концентрации иттрия до $x=0.15$ (рис. 3) в образце наблюдается смещение основного интерференционного максимума в сторону меньших углов, соответствующего фазе (201)-R, слившейся в единую группу с суммарным максимумом двух фаз (110)-R +(110)-O. Смещение дебаевской линии в сторону меньших углов при уменьшении ионного радиуса у элемента замещения $\mathrm{Y}^{3}$ вызвано увеличением растягивающих упругих напряжений в основной кристаллографической группе плоскостей, на что указывает и изменение 


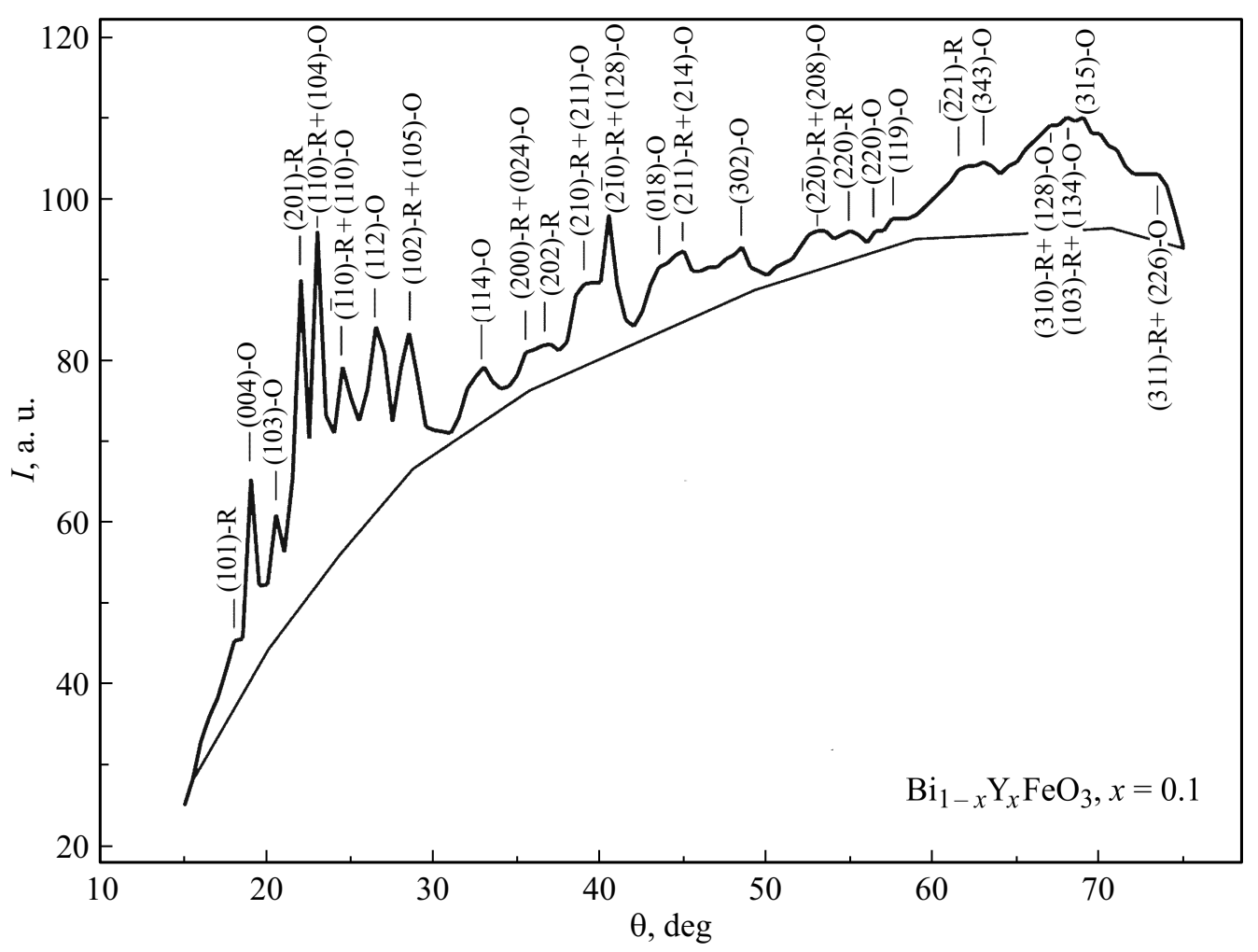

Рис. 2. Дифракционная картина от образца $\mathrm{Bi}_{1-x} \mathrm{Y}_{x} \mathrm{FeO}_{3}$ при $x=0.1$.

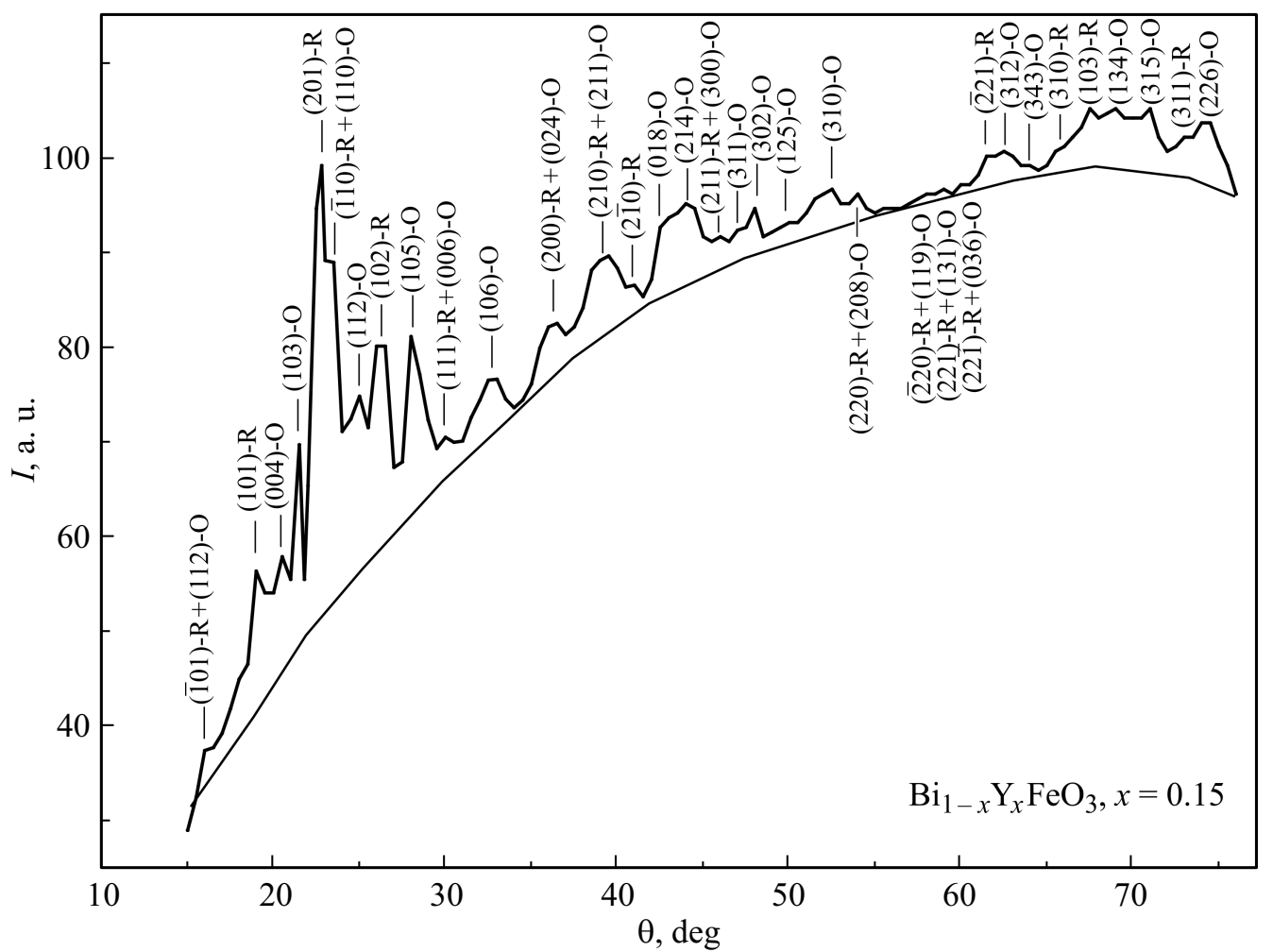

Pис. 3. Дифракционная картина от образца $\mathrm{Bi}_{1-x} \mathrm{Y}_{x} \mathrm{FeO}_{3}$ при $x=0.15$. 


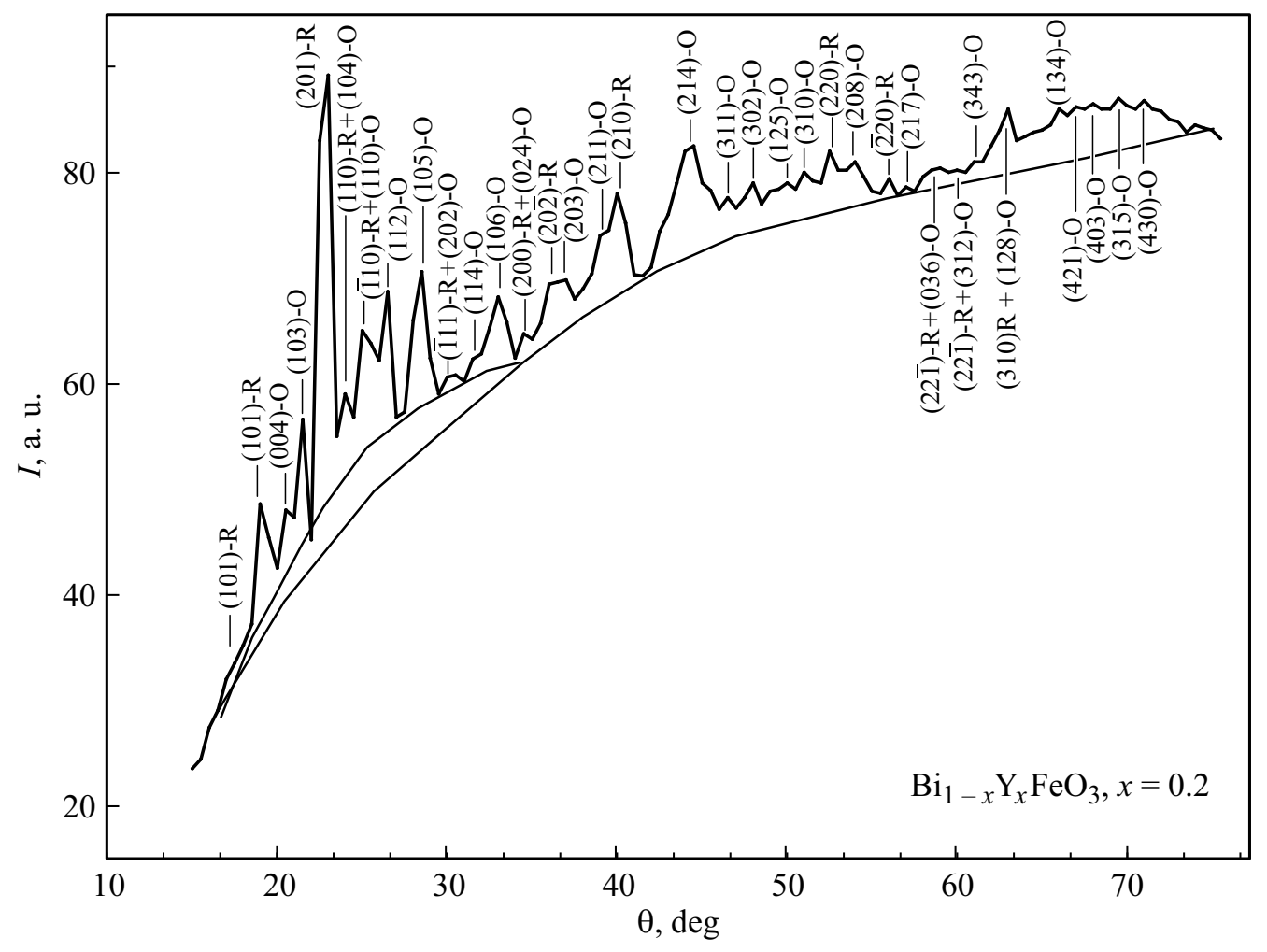

Рис. 4. Дифракционная картина от образца $\mathrm{Bi}_{1-x} \mathrm{Y}_{x} \mathrm{FeO}_{3}$ при $x=0.2$.

формы рядом расположенных линий дифракционного максимума на рис. 3 по сравнению с рис. 1 и 2. Происходит усиление фазового взаимодействия орторомбического и ромбоэдрического кристаллографического порядков, что сопровождается усилением локального беспорядка в виде аморфизации с проявлением диффузного рассеяния в форме гало (рис. 3 по сравнению с рис. 1).

Данная группа плоскостей (201)-R и (110)-R+(110)-O совместно с рядом расположенными (112)-O и (102)-R группами плоскостей сформировали аморфизацию описанных структурных групп, что проявляется в виде диффузных гало у оснований соответствующих линий.

При дальнейшем увеличении концентрации иттрия до $x=0.2$ в составе керамики (рис. 4) основной интерференционный максимум на дифракционной картине принадлежит ромбоэдрической фазе (201)-R.

Рядом расположены двухфазные отражения

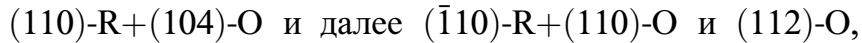
(105)-O, (106)-О, основания которых представляют гало, полученное в результате когерентного рассеяния рентгеновских лучей от низкоразмерных группировок атомов аморфизированной структуры, когда нарушен дальний атомный порядок во фрагментах кристаллизующихся плоскостей в процессе синтеза материала.

Для сравнения фазового состава изучаемых образцов мы провели двойную нормировку дифракционных отражений. При первой нормировке измеренные экспе- риментальные интенсивности линий в виде ординат от максимума до линии фона выписывали в таблицу. Затем самую интенсивную линию $\left(I_{0}\right)$ принимали за 100 , и находили первое нормировочное отношение $N_{1}=100 / I_{0}$, на значение которого умножали весь столбик значений экспериментальных интенсивностей. Таким образом, уравнивали дифракционные отражения для каждого из экспериментальных образцов. Далее нормированные интенсивности расписывали в два новых столбца по фазовой принадлежности ( $\mathrm{R}$ или $\mathrm{O})$, при наложении отражений значение делилось поровну на две фазы. Разделенные по фазовой принадлежности интенсивности суммировали в каждом столбце, и записывали полученные суммы отдельно для каждой фазы. Находили общую сумму, которую принимали за 100\% и затем вычисляли второй нормирующий множитель $N_{2}$, который позволяет оценить концентрацию каждой из присутствующих в образце фаз.

На рис. 5 показана зависимость изменений фазового состава исследованных образцов $\mathrm{Bi}_{1-x} \mathrm{Y}_{x} \mathrm{FeO}_{3}$ при $x=0$, $0.1,0.15,0.2$. Образцы $\mathrm{Bi}_{1-x} \mathrm{Y}_{x} \mathrm{FeO}_{3}$ имеют двухфазную структуру во всем интервале концентраций $x=0,0.1$, $0.15,0.2$.

При этом показано, что при $x=0-0.15$ концентрация орторомбической фазы чуть больше 50\% и слабо увеличивается, а ромбоэдрической - чуть меньше $50 \%$ и слабо уменьшается в интервале $x=0-0.15$; а затем концентрация орторомбической фазы резко изменяются 


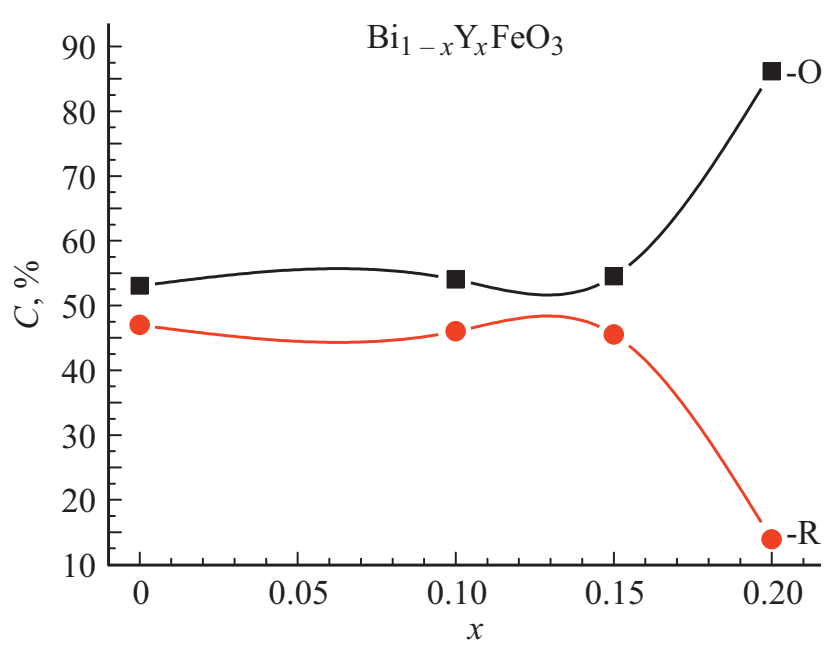

Рис. 5. Зависимость изменений фазового состава образцов $\mathrm{Bi}_{1-x} \mathrm{Y}_{x} \mathrm{FeO}_{3}$ при $x=0,0.1,0.15,0.2$.

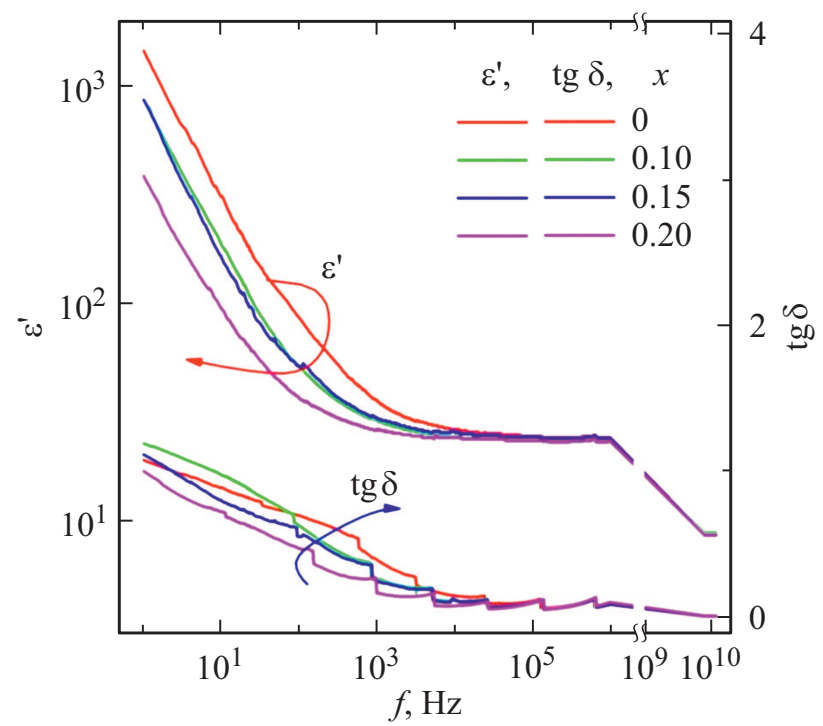

Рис. 6. Частотная зависимость диэлектрической проницаемости $\varepsilon^{\prime}(f)$ и тангенса угла диэлектрических потерь $\operatorname{tg} \delta(f)$ керамики $\mathrm{Bi}_{1-x} \mathrm{Y}_{x} \mathrm{FeO}_{3}$.

до $87 \%$ и до $13 \%$ для ромбоэдрической фазы. Этот результат указывает на выявленный концентрационный фазовый переход $\mathrm{R} \rightarrow \mathrm{O}$ в $\mathrm{Bi}_{1-x} \mathrm{Y}_{x} \mathrm{FeO}_{3}$ при $x=0.2$. Выявленная нами закономерность согласуется с опубликованными результатами по изменению намагниченности подобных образцов, полученными в [10], в которой показано, что намагниченность насыщения с изменением концентрации иттрия имеет постепенно увеличивающиеся значения, $(0.7468,2.1108,3.3276) \mathrm{emu} / \mathrm{g}$ для $x=0.05,0.10,0.15$ и затем более крутая зависимость, соответствующая величине $6.2703 \mathrm{emu} / \mathrm{g}$ для $x=0.20$.

Из результатов исследований диэлектрических параметров образцов (рис. 6) видно, что значение диэлектрической проницаемости керамики $\mathrm{Bi}_{1-x} \mathrm{Y}_{x} \mathrm{FeO}_{3}$ изменяется от $\varepsilon^{\prime} \sim 10^{2}$ в НЧ диапазоне до $\varepsilon^{\prime} \sim 8.5$ в СВЧ диапазоне. На рис. 6 показано, что дисперсия диэлектрической проницаемости имеет релаксационный характер. Все исследованные составы $\mathrm{Bi}_{1-x} \mathrm{Y}_{x} \mathrm{FeO}_{3}$ при $x=0,0.1,0.15,0.2$ имеют высокие значения диэлектрической проницаемости $\varepsilon^{\prime} \sim(3.8-15) \cdot 10^{2}$ на частоте $1 \mathrm{~Hz}$, которые уменьшаются до $\varepsilon^{\prime} \sim 23,5$ с увеличением частоты до $1 \mathrm{MHz}$; тангенс угла диэлектрических потерь также уменьшается от значения $\operatorname{tg} \delta \sim 1.07$ на частоте $1 \mathrm{~Hz}$ до $\operatorname{tg} \delta \sim 0.08$ на частоте $1 \mathrm{MHz}$.

Высокие потери на низкой частоте $\sim 1 \mathrm{~Hz}$ свидетельствуют о наличии в исследуемом материале миграционной поляризации, что, согласно $[11,12]$, проявляется как увеличение проводимости. В СВЧ диапазоне значение диэлектрической проницаемости уменьшается до $\varepsilon_{\infty} \sim 8.5$, а тангенс угла диэлектрических потерь уменьшается до $\sim 2 \cdot 10^{-3}$ для всех исследуемых составов $\mathrm{Bi}_{1-x} \mathrm{Y}_{x} \mathrm{FeO}_{3}$, кроме образца $\mathrm{Bi}_{0.85} \mathrm{Y}_{0.15} \mathrm{FeO}_{3}$, для которого потери на порядок ниже $-\operatorname{tg} \delta \sim 4 \cdot 10^{-4}$.

\section{Выводы}

В результате исследования изменения концентрации иттрия в составе образцов $\mathrm{Bi}_{1-x} \mathrm{Y}_{x} \mathrm{FeO}_{3}$ при $=0,0.1$, $0.15,0.2$ были установлены следующие закономерности.

1. При $x=0.15-0.2$ обнаружен концентрационный фазовый переход ромбоэдрической структуры в орторомбическую с резким изменением их концентраций от равновеликих, близких к 50\%, до разновеликих (87\% для орторомбической и $13 \%$ ромбоэдрической фаз).

2. Выявлена миграционная поляризация в образцах $\mathrm{Bi}_{1-x} \mathrm{Y}_{x} \mathrm{FeO}_{3}$ при $x=0,0.1,0.15,0.2$ при относительно высоких потерях на низкой частоте $\sim 1 \mathrm{~Hz}$.

\section{Конфликт интересов}

Авторы заявляют, что у них нет конфликта интересов.

\section{Список литературы}

[1] W. Eerenstein, N.D. Mathur, J.F. Scott. Nature, 442, 759 (2006).

[2] А.Т. Козаков, К.А. Гуглев, В.В. Илясов, И.В. Ершов, А.В. Никольский, В.Г. Смотраков, В.В. Еремкин. ФТТ, 53 (1), 41 (2011).

[3] A.A. Belik. J. Solid Stat. Chem., 195, 32 (2012).

[4] M. Azuma, H. Kanda, A.A. Belik, Y. Shimakawa, M. Takano. J. Magn. Magn. Mater., 310, 1177 (2007).

[5] N.A. Liedienov, A.V. Pashchenko, V.A. Turchenko, V.Ya. Sycheva, A.V. Voznyak, V.P. Kladko, A.I. Gudimenko, D.D. Tatarchuk, Y.V. Didenko, I.V. Fesych, I.I. Makoed, A.T. Kozakov, G.G. Levchenko. Ceram. Int., 45 (12), 14873 (2019).

[6] A.V. Pashchenko, N.A. Liedienov, Q. Li, et al. // J. Magn. Magn. Mater., 2019. Vol. 483. P. 100-113.

[7] 3.А. Самойленко, Н.Н. Ивахненко, Е.И. Пушенко, Е.И. Шемченко, В.Н. Варюхин. ЖТФ, 90 (2), 318 (2020). 
[8] D.D. Tatarchuk. Proc. of the 35th Int. Sci. Conf. Electronics and Nanotechnology (ELNANO-2015). Kyiv, Ukraine, 231 (2015).

[9] М.А. Кривоглаз. Дифбузное рассеяние рентгеновских лучей и нейтронов на фллктууационных неоднородностях в неидеальных кристаллах (Наук. думка, Киев, 1984), $281 \mathrm{c}$.

[10] B. Feng, H. Xue, Zh. Xiong. Chin. Sci. Bull., 55, 452 (2010).

[11] Y.V. Didenko. Proc. of the 34th Int. Sci. Conf. Electronics and Nanotechnology (ELNANO-2014). Kyiv, Ukraine, 73 (2014).

[12] I.I. Makoed, N.A. Liedienov, A.V. Pashchenko, G.G. Levchenko, D.D. Tatarchuk, Y.V. Didenko, A.A. Amirov, G.S. Rimskiy, K.I. Yanushkevich. J. Alloy. Compd., 155859, 842 (2020). 